Revista de BIOLOGía Tropical

\title{
Bibliometría de la colaboración e impacto de la Revista de Biología Tropical (Web of Science 2003-2012)
}

\author{
Daniela De Filippo ${ }^{1,2,3}$, Saray Córdoba González ${ }^{4}$ \& Elías Sanz-Casado ${ }^{1,2,3}$ \\ 1. Laboratorio de Estudios Métricos de la Información (LEMI). Universidad Carlos III de Madrid. C/ Madrid 126, Getafe \\ 28903, Madrid, España; dfilippo@bib.uc3m.es, elias@bib.uc3m.es \\ 2. Research Institute for Higher Education and Science (INAECU). Madrid, España \\ 3. Unidad Asociada IFS (CSIC)-LEMI (UC3M), Madrid, España \\ 4. Universidad de Costa Rica. San José, Costa Rica; saraycg@gmail.com
}

Recibido 28-X-2014. Corregido 03-VIII-2015. Aceptado 03-IX-2015.

\begin{abstract}
Bibliometry of collaboration and impact of the Revista de Biología Tropical (Web of Science 2003-2012). The activity analysis of a scientific journal is relevant to know the evolution of its characteristics over time. In this paper, results of a bibliometric study of the Revista de Biología Tropical/International Journal of Tropical Biology and Conservation (Costa Rica) are presented. The goal of this study was to describe the main characteristics of its scientific production, and analyze its level of collaboration and its impact between the years 2003-2012. Data was derived from the Web of Science (Thomson-Reuters), and the relationship among authors and coauthors, institutions and countries, and their links with the citations received were analyzed for that period. Descriptive statistics about production (number of documents per year, institution and country), collaboration (authorship index, collaboration among institutions and countries) and impact (IF, position in JCR and number of citations received) were collected. Results showed that the journal has published 1473 papers in this period, in similar proportions English and Spanish. Mexico, Costa Rica, Venezuela and Colombia are the most common countries of origin, with the Universidad of Costa Rica, Universidad Autónoma de Mexico and the University of Puerto Rico as the most common leader institutions. Collaboration between authors, institutions and countries has shown an increasing trend over the last decade. The co-author index was 3.07 per document, $63 \%$ of publications included 2 or more institutions, and $22 \%$ of the papers were product of international collaboration. The most common collaboration link was between Costa Rica and the United States of America. The impact factor has been oscillating during this last decade, reaching a maximum in 2012 (IF JCR $=0.553$ ). Besides, $10 \%$ of the most cited papers concentrated half of the citations received by the journal, and have a very high number of citations, compared with the journal mean. The main countries that cite the journal were USA, Brazil, Mexico, Argentina and Costa Rica. Data showed an increasing collaboration between authors, institutions and countries, and a direct relationship between the increase of this collaboration and the received impact. Rev. Biol. Trop. 64 (1): 147-156. Epub 2016 March 01.
\end{abstract}

Palabras clave: bibliometry, International Journal of Tropical Biology and Conservation, scientific collaboration, Costa Rica, impact factor.

Costa Rica es, a pesar de su reducido tamaño, uno de los países más productivos de América Latina, alcanzando en la última década la décima posición en Latinoamérica por producción científica en la Web of Science (Thomson-Reuters, 2014a) (WoS). La importancia de la actividad científica se evidencia también en el número de investigadores que trabajan en sus universidades y centros de investigación. Según la RICYT, a este corresponde a 2.9 en equivalente jornada completa por cada 1000 integrantes de la población económicamente activa. Estas cifras lo ubican en la primera posición de América Latina, junto a Argentina, y por encima de Brasil, Chile y México (RICYT, 2014). 
Considerando las publicaciones científicas según grandes áreas temáticas, el país presenta una clara especialización en Ciencias de la Vida (40\% de su producción). Dentro de este campo destaca la categoría Biology con el 9 $\%$ de la producción del país en la WoS (SanzCasado, De Filippo, \& Marugán, 2014). Esta especialización está íntimamente vinculada a condiciones geográficas y climatológicas que hacen de Costa Rica uno de los países con mayor biodiversidad. Por esta razón, Costa Rica se ha convertido en uno de los líderes mundiales en la investigación sobre conservación de la biodiversidad tropical y reconocida en el mundo por su desarrollo en esta área.

Uno de los principales medios de difusión para los resultados de la investigación en este campo ha sido la Revista de Biología Tropical, una publicación de la Universidad de Costa Rica (UCR) que se edita con periodicidad trimestral, desde 1953. A partir de su inclusión en WoS -en 1969- ha sido la única publicación centroamericana indexada en Science Citation Index (SCI) y una de las pocas de Latinoamérica. Así, en la categoría Biology únicamente se encuentran incluidas en la versión 2013 del Journal Citation Reports, seis revistas de la región: Biocell de Argentina, Biological Research de Chile, y las brasileñas: Bioscience Journal, Brazilian Archives of Biology and Technology, Brazilian Journal of Biology, y Brazilian Journal of Medical and Biological Research.

Desde el punto de vista bibliométrico, el interés por esta revista se ha evidenciado al ser estudiada por diferentes autores (Monge-Nájera \& Díaz, 1988; Cortés \& Nielsen-Muñoz, 2002; Monge-Nájera \& Nielsen-Muñoz, 2005; Nielsen-Muñoz, Azofeifa-Mora, \& MongeNájera, 2012). En este caso, se presentan las características de la producción científica más reciente publicada en la revista (2003-2012). El principal objetivo es profundizar en el estudio de dos variables que consideramos centrales en el proceso de producción de conocimiento: la colaboración y la citación. Numerosos autores han mencionado la importancia creciente de la colaboración, especialmente entre países aunque con diferencias entre áreas temáticas y disciplinas (Beaver \& Rosen, 1979a, 1979b; Wagner-Döbler, 2001; Wagner \& Leydesdorff, 2005; Gazni, Sugimoto, \& Didegah, 2012). También la relación entre colaboración e impacto ha sido detectada en estudios previos (Bridgstock, 1991; van Raan, 1998; Persson, Glänzel, \& Dannell, 2004, Gazni et al., 2012) aunque no se ha constatado en todos los campos. Por tanto, en este trabajo se analizan los patrones de colaboración e impacto de la Revista de Biología Tropical para verificar si existe alguna relación entre ambas variables.

\section{MATERIALES Y MÉTODOS}

Se ha realizado un estudio de la Revista de Biología Tropical durante el período 20032012 con métodos bibliométricos tal como es habitual para el análisis de la producción de una revista científica (Ríos-Hilario \& TraviesoRodríguez, 2013; Sanz-Velero, Tomás-Casterá, \& Tomás-Gorriz, 2014).

Para ello, se ha utilizado la base de datos Web of Science de Thomsom Reuters (consulta realizada en marzo 2014). A pesar de las limitaciones de esta fuente (Gómez-Caridad \& Bordons-Gangas, 1996) se ha utilizado porque presenta la dirección de todos los autores y esto permite realizar estudios de colaboración. Brinda, además, datos sobre el impacto de cada documento. Las publicaciones se recogieron con la estrategia "SO = Revista de Biología Tropical AND PY = 2003-2012" se identificó toda la producción del período analizado y se consideraron todas las tipologías documentales publicadas. Los registros fueron descargados y se construyó una base de datos relacional en MySQL para el tratamiento de la información, posteriormente fueron normalizados y se codificaron las direcciones de instituciones y países. Así se obtuvieron los siguientes indicadores:

Producción: Número de documentos publicados por año utilizando el recuento total (Pravdic \& Olluic-Vucovic, 1991) -tal y como lo realiza la WoS-, su tipología documental e idioma. 
Colaboración: Se han analizado los datos del campo address considerando las instituciones y países firmantes de las publicaciones. En 45 documentos no se presentó información sobre la filiación institucional de los autores por lo que se consultó el campo reprint address, de donde se obtuvo la dirección de contacto del autor principal y esta se añadió. Así, se han podido completar 12 registros quedando otros 33 sin información. Los indicadores obtenidos fueron: -Índice de co-autoría (número de firmas por documento), -colaboración institucional (número de centros firmantes), -colaboración internacional (número de países por documento). La representación gráfica de las redes de colaboración internacional se realizó con el programa de visualización Gephi (0.8.2) utilizando el algoritmo Fruchterman y Reingold (1991).

Impacto: Factor de Impacto (FI) de la revista por año -obtenido a través del JCR-, citas recibidas por documento y año, porcentaje de documentos no citados, H-Index, documentos más citados; países, instituciones y revistas citantes.

\section{RESULTADOS}

Producción: Entre 2003 y 2012 se recogieron 1473 publicaciones de la Revista de Biología Tropical en la WoS, y es el año 2008 el de mayor número de documentos (Fig. 1). En cuanto al idioma, la revista publica proporciones similares de documentos en español (50\%) y en inglés (49\%).

Al considerar la región de origen de los autores, la mayoría corresponde a centros Latinoamericanos, concretamente 1362 documentos $(75 \%)$, seguidos de Norte América, con 229 documentos (12.7\%) y Europa, con 138 documentos $(7.7 \%)$. En cuanto a la distribución por países, México es el que cuenta con mayor número de publicaciones en la década estudiada, seguido de Costa Rica con un porcentaje muy similar, y Estados Unidos en tercer lugar (Apéndice digital, Fig. 1).

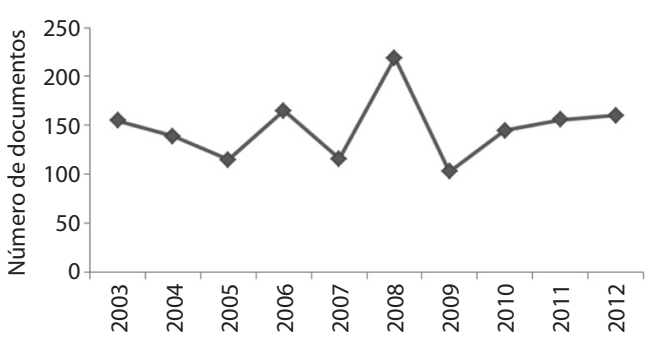

Fig. 1. Número de documentos publicados por año.

Fig. 1. Number of papers published per year.

Entre las instituciones más productivas destacan las universidades y los centros de investigación de América Latina. En este sentido, la Universidad de Costa Rica aparece en primera posición con un $17 \%$ de los documentos publicados. Le siguen la Universidad Nacional Autónoma de México, la Universidad de Puerto Rico, y la Universidad de Oriente de Venezuela. Las primeras instituciones que aparecen en la lista, fuera de la región latinoamericana, son de Estados Unidos (Florida State University) y de España (Consejo Superior de Investigaciones Científicas) (Apéndice digital, Cuadro 1).

Colaboración: Considerando la colaboración entre autores en los diez años analizados, el promedio del índice de co-autoría de la Revista de Biología Tropical ha sido de 3.07 firmas por documento. Entre 2003 y 2012 estos valores han aumentado de 2.43 a 3.46. Los documentos de autoría única han sido 223 (un $15 \%$ del total en los 10 años) y han ido decreciendo del $3 \%$ en 2003 al $0.95 \%$ en 2012 . Se han detectado documentos firmados hasta por 31 autores, aunque los más numerosos han sido los de tres firmas. En cuanto al número de centros firmantes, en promedio el $37.45 \%$ de los documentos ha sido elaborado por una única institución (el $54 \%$ en 2003 y el $31 \%$ en 2012). Los documentos firmados por dos instituciones representan el $35.14 \%$, mientras que los de tres instituciones alcanzaron el $17.16 \%$. Hay un solo caso de 16 instituciones que firman una publicación, pero se trata de una colaboración aislada. Por otro lado, los documentos firmados 


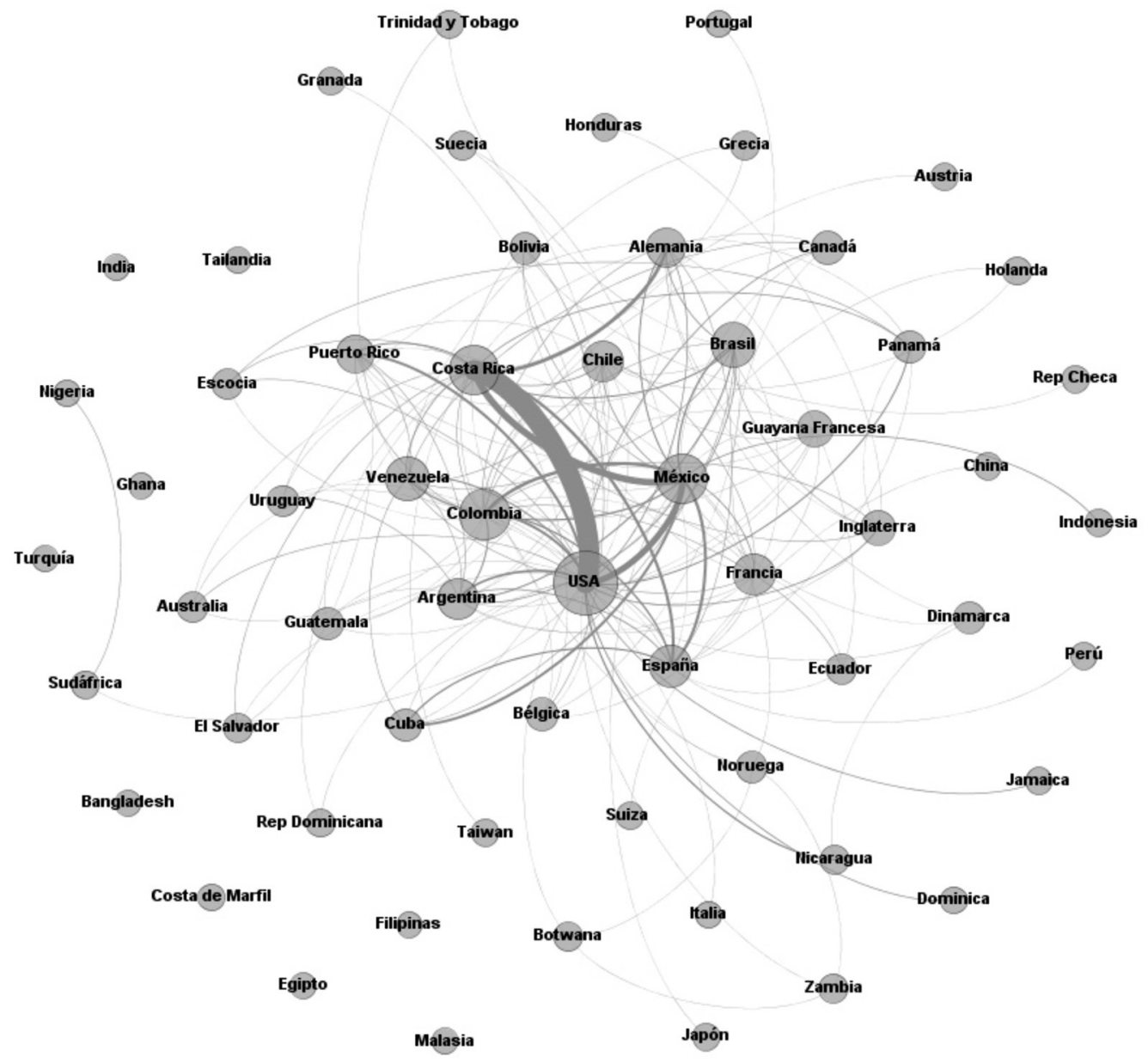

Fig. 2. Red de colaboración entre países firmantes.

Fig. 2. Network collaboration between signatory countries.

por una institución pertenecen principalmente a centros de México (117 doc.) y Costa Rica (91 doc.), seguidos de Colombia (53 doc.), Venezuela (50 doc.) y Estados Unidos (43 doc.). En cuanto al número de países firmantes, un $78 \%$ de las publicaciones han sido realizadas por investigadores de un único país, es decir, sin colaboración internacional. Estos porcentajes han descendido del $84 \%$ en 2003 al $80 \%$ en 2012 (Apéndice digital, Fig. 2). Entre los documentos en colaboración internacional, los más frecuentes son los realizados entre dos países $(16.84 \%)$. En el cuadro 1 se puede observar el número de firmas, instituciones y países por documento y se destaca que en todos los casos la colaboración ha aumentado entre 2003 y 2012. (Cuadro 1).

Las relaciones de colaboración entre países se muestran en la figura 2. Los nodos representan a cada país que ha firmado alguna publicación durante el período de estudio y las aristas muestran los vínculos entre ellos. El tamaño de los nodos indica el número de colaboradores de cada país y con ello, el grado de colaboración. De ahí se puede destacar que la mayor frecuencia de colaboración (líneas más gruesas) se produce entre Costa Rica y Estados Unidos, siendo México un colaborador 
CUADRO 1

Colaboración entre autores, instituciones y países

TABLE 1

Collaboration between authors, institutions and countries

\begin{tabular}{cccccccc} 
Año & N doc & Firmas & Firmas/doc & Instituciones & Inst/doc & País & Países/doc \\
2003 & 155 & 376 & 2.43 & 247 & 1.59 & 176 & 1.14 \\
2004 & 139 & 389 & 2.80 & 278 & 2.00 & 167 & 1.20 \\
2005 & 115 & 336 & 2.92 & 191 & 1.66 & 134 & 1.17 \\
2006 & 165 & 428 & 2.59 & 297 & 1.80 & 194 & 1.18 \\
2007 & 116 & 334 & 2.88 & 202 & 1.74 & 141 & 1.22 \\
2008 & 219 & 756 & 3.45 & 482 & 2.20 & 290 & 1.32 \\
2009 & 103 & 341 & 3.31 & 212 & 2.06 & 127 & 1.23 \\
2010 & 145 & 474 & 3.27 & 287 & 1.98 & 174 & 1.20 \\
2011 & 156 & 541 & 3.47 & 326 & 2.09 & 184 & 1.18 \\
2012 & 160 & 553 & 3.46 & 351 & 2.19 & 197 & 1.23 \\
Total & 1473 & 4528 & 3.07 & 2873 & 1.95 & 1784 & 1.21 \\
\hline
\end{tabular}

habitual de estos dos países. Aquellos nodos que permanecen aislados (Malasia, Ghana, Filipinas, India, Egipto, Turquía, Tailandia, Bangladesh y China) corresponden a países que no han firmado documentos en colaboración. El grado medio de la red es de 5.3, siendo Estados Unidos, Colombia, Costa Rica y México los países con mayor grado (nodos de mayor tamaño) (Fig. 2).

Impacto: Se han analizado los indicadores relacionados con el FI de la Revista de Biología Tropical y su posición en el JCR (Thomson Reuters, 2014b). Dentro de la categoría Bio$\log y$, la revista ha sufrido oscilaciones anuales con un valor mínimo de 0.199 (en 2008) y un máximo de 0.553 (en 2012). Aunque siempre ha permanecido en el cuarto cuartil, se ha calculado la posición normalizada en función del total de revistas en su WoS category para cada año; esto es el cociente entre la posición alcanzada sobre el total de revistas de esa categoría. Así, se observa que las mejores posiciones han sido obtenidas en los años 2003 y 2012, con los valores normalizados más altos, mientras que en 2008 ha tenido la posición más baja (Cuadro 2).

Con respecto a las citas de los 1473 documentos analizados, se destaca que estos han recibido 3101 citas desde el momento de su publicación hasta marzo 2014 (fecha de recogida de los datos). Si bien el promedio ha sido de 2.11 citas por documento, hay que recordar que, al tratarse de valores acumulativos, los más antiguos son los que tienen más posibilidades de ser citados. Las publicaciones del 2008 son las que recibieron mayor número de citas y esto coincide con que en esa fecha se publicaron, excepcionalmente, 4 suplementos (lo habitual son 2 suplementos anuales). El porcentaje de documentos NO citados ha mostrado un promedio del $37.7 \%$ (Cuadro 3 ).

\section{CUADRO 2}

Factor de impacto y posición en JCR

TABLE 2

Impact factor and position in JCR

\begin{tabular}{ccccc} 
Año & Posición & $\begin{array}{c}\text { Valor } \\
\text { Norm }\end{array}$ & FI & $\begin{array}{c}\text { FI (median) } \\
\text { Biology }\end{array}$ \\
\hline 2003 & $53 / 65$ & 0.18 & 0.354 & 0.971 \\
2004 & $58 / 64$ & 0.09 & 0.220 & 1.105 \\
2005 & $58 / 65$ & 0.11 & 0.322 & 1.179 \\
2006 & $60 / 65$ & 0.08 & 0.217 & 1.138 \\
2007 & $65 / 71$ & 0.08 & 0.274 & 1.292 \\
2008 & $71 / 72$ & 0.01 & 0.199 & 1.383 \\
2009 & $71 / 76$ & 0.07 & 0.329 & 1.337 \\
2010 & $73 / 86$ & 0.15 & 0.435 & 1.339 \\
2011 & $73 / 85$ & 0.14 & 0.459 & 1.540 \\
2012 & $68 / 83$ & 0.18 & 0.553 & 1.458 \\
\hline
\end{tabular}


CUADRO 3

Citas recibidas por año

TABLE 3

Citations per year

\begin{tabular}{ccccc} 
Años & Citas & Citas/doc & $\begin{array}{c}\text { Doc } \\
\text { NO citados }\end{array}$ & $\%$ \\
\hline 2003 & 520 & 3.35 & 40 & 25.81 \\
2004 & 496 & 3.57 & 27 & 19.42 \\
2005 & 406 & 3.53 & 22 & 19.13 \\
2006 & 381 & 2.31 & 57 & 34.55 \\
2007 & 297 & 2.56 & 27 & 23.28 \\
2008 & 471 & 2.15 & 77 & 35.16 \\
2009 & 149 & 1.45 & 38 & 36.89 \\
2010 & 229 & 1.58 & 51 & 35.17 \\
2011 & 115 & 0.74 & 85 & 54.49 \\
2012 & 37 & 0.23 & 132 & 82.50 \\
Total & 3101 & 2.11 & 556 & 37.75 \\
\hline
\end{tabular}

El análisis de citas muestra que los principales países citantes son: Estados Unidos (21 $\%$ de los documentos citantes), Brasil (18\%), México (17\%), Argentina (7\%) y Costa Rica (7\%). Las instituciones que más han citado a la Revista de Biología Tropical fueron la Universidad Nacional Autónoma de México, la Universidad de Costa Rica, el Consejo Nacional de Investigaciones Científicas y Técnicas (Conicet, Argentina), la Universidade de São Paulo y el Instituto Politécnico Nacional de México. En el Apéndice digital, Cuadro 2 se presentan las principales revistas citantes entre las que destaca la propia Revista de Biología Tropical (12\%), seguida -aunque con un porcentaje menor- de Zootaxa y PLOS One. (Apéndice digital, Cuadro 2).

$\mathrm{Al}$ establecer la relación entre la producción y la citación se aprecia que la Revista de Biología Tropical cuenta con un H-Index de 16 , es decir, que ha publicado 16 documentos con al menos 16 citas. Por otra parte, cuando se analiza el $10 \%$ de los documentos más citados (147) se observa que ha recibido 1620 citas, un $52 \%$ de las citas de todos los documentos publicados. Esto se traduce en un ratio de 11.02 citas por documento, con un máximo de 31 citas y un mínimo de 7 . Entre ellos, un $25 \%$ ha sido firmado por centros de Estados Unidos, un
$22 \%$ por instituciones de México y un $21 \%$ por centros costarricenses. En estos documentos el porcentaje de colaboración internacional es mayor que el del promedio de la revista $(22 \%)$ (Cuadro 4).

\section{DISCUSIÓN}

En líneas generales, se observa que en la década analizada ha existido un número estable de documentos por año, predominando el tipo "artículos". Esta estabilidad refleja el interés que tiene la revista en la comunidad científica,

\section{CUADRO 4}

Distribución de la producción y colaboración internacional en el $10 \%$ de los documentos más citados

TABLE 4

Production and distribution of international collaboration in the $10 \%$ most cited papers

\begin{tabular}{lcccc}
\hline \multirow{2}{*}{\multicolumn{1}{c}{ País }} & \multicolumn{2}{c}{ Producción } & \multicolumn{2}{c}{$\begin{array}{c}\text { Colaboración } \\
\text { Internacional }\end{array}$} \\
\cline { 2 - 5 } & $\mathrm{N}$ doc & $\%$ & $\mathrm{~N}$ doc & $\%$ \\
\hline Estados Unidos & 37 & 25.17 & 13 & 35.1 \\
México & 33 & 22.45 & 8 & 24.2 \\
Costa Rica & 31 & 21.09 & 9 & 29.0 \\
Brasil & 22 & 14.97 & 3 & 13.6 \\
Argentina & 9 & 6.12 & 4 & 44.4 \\
Alemania & 7 & 4.76 & 4 & 57.1 \\
Venezuela & 7 & 4.76 & 3 & 42.9 \\
Colombia & 5 & 3.40 & 0 & 0.0 \\
Chile & 4 & 2.72 & 2 & 50.0 \\
India & 4 & 2.72 & 0 & 0.0 \\
Cuba & 2 & 1.36 & 2 & 100.0 \\
Guatemala & 2 & 1.36 & 2 & 100.0 \\
Nigeria & 2 & 1.36 & 2 & 100.0 \\
Panamá & 2 & 1.36 & 1 & 50.0 \\
France & 1 & 0.68 & 1 & 100.0 \\
Jamaica & 1 & 0.68 & 1 & 100.0 \\
Japón & 1 & 0.68 & 1 & 100.0 \\
Perú & 1 & 0.68 & 0 & 0.0 \\
España & 1 & 0.68 & 1 & 100.0 \\
Suecia & 1 & 0.68 & 1 & 100.0 \\
Trinidad y Tobago & 1 & 0.68 & 1 & 100.0 \\
Turquía & 1 & 0.68 & 0 & 0.0 \\
Estados Asociados Antillas & 1 & 0.68 & 1 & 100.0 \\
\hline & & & & \\
& & 1.36
\end{tabular}


ya que asegura un flujo regular y constante de manuscritos para su publicación.

En cuanto al idioma, la mitad de los documentos fueron publicados en español, tendencia que se ha mantenido constante desde la creación de la revista, tal como se muestra en trabajos anteriores (Monge-Nájera \& Díaz, 1998). El análisis de estas cifras puede resultar interesante considerando que el inglés es la lengua dominante en las revistas de ciencias de la vida indexadas en bases de datos internacionales. En este sentido, estudios recientes muestran que este idioma representa el $89 \%$ en SCI, el $72 \%$ en Biosis y el $58 \%$ en CAB (Michán \& Llorente-Bousquets, 2010). Esta característica lingüística de la producción de la Revista de Biología Tropical está muy vinculada con su temática que resulta de interés especialmente para investigadores de regiones tropicales que, por lo general, son de habla hispana o lusófona -que suelen leer con facilidad los textos en español debido a las similitudes idiomáticas- tal como mencionan NielsenMuñoz et al. (2012).

Es quizá esta especialización temática -muy vinculada con determinadas regiones geográficas - la que determina el ámbito de influencia de la revista. El estudio de la afiliación de los autores, en función de su centro de adscripción muestra una mayor contribución de países latinoamericanos especialmente de México, Costa Rica, Venezuela o Colombia. La presencia destacada de Costa Rica se puede explicar por diversos motivos: en primer lugar, es el país de origen de la revista que, al tratarse de la única publicación indizada en SCI, se convierte en un órgano de difusión central para los investigadores del campo. A esto se suma la relevancia que el área tiene para el país, ya que biología y ambiente son los puntos fuertes en los que se ha especializado Costa Rica (Fernández, Sancho, Morillo, De Filippo, \& Gómez, 2005). La importancia de la revista para los investigadores costarricenses se evidencia también al constatar que es la revista con mayor producción del país y que concentra un $8 \%$ de los documentos publicados en todas las áreas del conocimiento (Sanz-Casado et al., 2014).
Es relevante mencionar también que esta presencia tan notoria de las publicaciones de Costa Rica en la revista ha sido constante desde la década del 50, como han mostrado estudios previos en los que aparece como el país mayoritariamente predominante (Monge-Nájera \& Díaz, 1998). Sin embargo, aunque en la actualidad la revista siga presentando una importante participación de investigadores costarricenses, la creciente presencia de autores extranjeros puede considerarse como un signo de apertura y reconocimiento por parte de la comunidad internacional. En este sentido, la inclusión de autores de instituciones de otros países contribuirá a aumentar el número de citaciones que reciba la revista de otras fuentes, con lo que disminuiría su porcentaje de autocitación.

En cuanto a las instituciones, la Universidad de Costa Rica ha sido la más destacada pues concentra un $17 \%$ de los documentos publicados en la revista en la década analizada. Además de ser el organismo editor, la alta participación de la UCR puede explicarse por tratarse de la institución más visible internacionalmente con una producción que alcanza el 50 $\%$ de los documentos del país en SCI (Lomonte \& Ainsworth, 2002; Sanz-Casado et al., 2014). Asimismo, dentro de la universidad, existen centros punteros en investigación como el Centro de Investigación en Biología Celular y Molecular (CIBCM), la Escuela de Biología y el Centro de Investigación en Ciencias del Mar y Limnología (CIMAR) -todos especializados en diversas áreas de la Biología- que son también los que cuentan con mayor financiación para proyectos de investigación (Arellano \& Jensen, 2006) y están entre los más productivos de la Revista de Biología Tropical (NielsenMuñoz et al., 2012).

Uno de los puntos centrales del presente estudio ha sido el análisis de la colaboración entre los autores. Los resultados muestran que el índice de co-autoría se ha incrementado pasando de 2.43 autores por documento en 2003 a 3.07 en 2012. Este crecimiento es producto de la reducción del número de artículos de autoría única que ha ido decreciendo en la década estudiada y representa en todo el período un 
$15 \%$ de la producción total; estas cifras son inferiores a las presentadas unos años antes por estudios como el de Nielsen-Muñoz et al. (2012), el cual mostraba un $22.6 \%$. La colaboración entre instituciones ha sido de un $62.5 \%$, es decir, que casi dos tercios de la producción ha sido firmada por autores pertenecientes a más de un centro. A pesar de la importancia creciente que actualmente tiene la colaboración científica entre autores e instituciones (Gazni et al., 2012), en este trabajo se ha observado que las publicaciones en colaboración internacional tienen una baja presencia en la revista. Aunque un $77 \%$ de los documentos han sido firmados por centros extranjeros, únicamente un $22 \%$ de las publicaciones tuvieron la firma de más de un país, dentro de las cuales, los vínculos más relevantes son los que se establecen entre centros de Costa Rica y Estados Unidos.

El impacto de la revista se ha analizado a través de su FI recogido en el JCR-SCI para la disciplina Biology. Como se pudo apreciar, los valores han ido en aumento logrando su máximo nivel en 2012. A pesar de este incremento, su posición es inferior a la mediana de la disciplina y ha permanecido hasta 2013 en el cuarto cuartil. Si bien estas posiciones resultan muy bajas, hay que considerar que dicha categoría sólo incluye 7 revistas de Latinoamérica; 5 de ellas en el último cuartil y otras dos en el tercero. Esto muestra que, ya sea por las temáticas o el idioma, las revistas de la región están lejos de ser punteras de su disciplina en términos de citación, lo cual se ha comentado en estudios previos sobre la producción iberoamericana (Rodríguez-Yunta, 2010).

En la década analizada, la Revista de Biología Tropical ha recibido en promedio 2.11 citas por documento y cuenta con un $37.7 \%$ de documentos no citados. Aunque es complejo realizar comparaciones de citación debido a la rapidez con que varían estos valores, para el mismo período se han detectado 6.36 citas/doc en la producción de la UCR en la categoría de Biology (Sanz-Casado et al., 2014). Esto podía mostrar que se trata de comunidades diferentes de investigadores: una cuyos hábitos de investigación están vinculados con temáticas más internacionales, así como con una mayor colaboración científica y otra comunidad más volcada hacia temas de investigación de interés regional y con menor colaboración científica, que es la que utilizaría la revista para divulgar sus resultados.

También se ha detectado que los documentos más citados tienen un porcentaje de colaboración internacional mayor que el promedio de publicaciones de la revista. Esto está en concordancia con los resultados obtenidos en otros estudios bibliométricos en los que se ha comprobado que la colaboración internacional contribuye al aumento de la visibilidad (Bridgstock, 1991; Persson et al, 2004; Adams, Black, Clemmons, \& Stephan, 2005) y por ende, a la citación. Por último, el análisis de consumo de la revista muestra que es la propia revista la que cita más sus artículos, mientras que si se consideran los países de las instituciones citantes destaca Estados Unidos, seguido de Brasil, México y Argentina. A pesar de las limitaciones de este estudio, de obtener citas únicamente de la $W o S$ (sin duda muchas otras se recogerían a través de revistas no indexadas en esta base de datos), esto pone de manifiesto que la Revista de Biología Tropical es un importante medio para investigadores de otros países, y que este uso y visibilidad de la revista, sin duda se incrementaría con la participación de un número mayor de autores extranjeros.

\section{RESUMEN}

El estudio de la actividad de una revista resulta esencial para conocer la evolución de sus características a lo largo del tiempo. En este trabajo se realiza un análisis bibliométrico de la Revista de Biología Tropical, utilizando como fuente la base de datos Web of Science (ThomsonReuters), entre los años 2003-2012. El objetivo del trabajo es describir las principales características de la producción de la revista y analizar el desarrollo de la colaboración y el impacto en la última década. Se estudia la colaboración entre autores, instituciones y países y su vinculación con las citas recibidas. Se han obtenido indicadores de producción (número de documentos por año, institución y país), colaboración (índice de co-autoría, colaboración entre centros y países) e impacto (factor de impacto, posición en el Journal Citation Report, citas recibidas). Los resultados muestran que en el período estudiado la revista ha publicado 1473 artículos. Predominan los documentos 
en inglés y español -por partes iguales-y los principales países de procedencia son México, Costa Rica, Venezuela y Colombia. La Universidad de Costa Rica es la institución que produce la mayor parte de los artículos, con un $17 \%$ de los documentos, seguida de la Universidad Autónoma de México y la Universidad de Puerto Rico. Se evidencia que la colaboración entre autores, instituciones y países ha crecido constantemente en la última década. El índice de coautoría ha sido de 3.07 autores por documento. Un $63 \%$ de las publicaciones se han realizado en colaboración entre 20 más instituciones y un $22 \%$ en colaboración internacional. Las relaciones de colaboración más frecuentes se producen entre Costa Rica y Estados Unidos. En cuanto al impacto de la revista, dentro de la categoría Biology el factor de impacto ha tenido oscilaciones, alcanzando su máximo valor en 2012 (FI JCR = 0.553). Se ha detectado que el $10 \%$ de los documentos más citados concentra la mitad de las citas recibidas por la revista y tienen un porcentaje de colaboración internacional muy superior a la media de la revista. Los principales países usuarios fueron Estados Unidos, Brasil, México, Argentina y Costa Rica, de quienes proviene la mayor citación de la revista. Los datos muestran una tendencia creciente a la colaboración entre autores, instituciones y países y una relación directa entre el incremento de esta colaboración y el impacto recibido.

Palabras clave: bibliometría, Revista de Biología Tropical, colaboración científica, factor de impacto, Costa Rica.

\section{REFERENCIAS}

Adams, J. D., Black, G. C., Clemmons, J. R., \& Stephan, E. (2005). Scientific teams and institutional collaborations: evidence from U.S. universities, 1981-1999. Research Policy, 34(3), 259-285.

Arellano, A. \& Jensen, H. (2006). Mapeando las redes de investigación en ciencias básicas de la Universidad de Costa Rica. Convergencia, 13, 181-213.

Beaver, D., \& Rosen, R. (1979a). Studies in Scientific Collaboration. Part II scientific co-autorship, research productivity and visibility in the French scientific elite (1799-1830). Scientometrics, 1(2), 133-149.

Beaver, D., \& Rosen, R. (1979b). Studies in Scientific Collaboration. Part III professionalization and the natural history of modern scientific co-autorship. Scientometrics, 1(3), 231-245.

Bridgstock, M. (1991). The quality of single and multiple authored papers. An unresolved problem. Scientometrics, 21(1), 37-48.

Cortés, J. \& Nielsen-Muñoz, V. (2002). Las ciencias del mar en la Revista de Biología Tropical en su 50 aniversario. Revista de Biología Tropical, 50(3-4), 903-907.

Fernández, M. T., Sancho, R., Morillo, F., De Filippo, D., \& Gómez, I. (2005). Indicadores de especialización temática de los países de América latina y el Caribe. In M. Albornoz \& D. Ratto (Eds.), Indicadores de Ciencia y Tecnología en Iberoamérica. Agenda 2005 (pp. 191-209). Buenos Aires, Argentina: RICYT.

Fruchterman, T. M. J., \& Reingold, E. M. (1991). Graph Drawing by Force-Directed Placement. Software: Practice and Experience, 21(11), 1129-1164.

Gazni, A., Sugimoto, C. R., \& Didegah, F. (2012). Mapping world scientific collaboration: Authors, Institutions, and Countries. Journal of the American Society for Information Science and Technology, 63(2), 323-335.

Gómez-Caridad, I. \& Bordons-Gangas, M. (1996). Limitaciones en el uso de los indicadores Bibliométricos para la evaluación científica. Política Científica, 46, 21-26.

Lomonte, B. \& Ainsworth, S. (2002). Publicaciones científicas de Costa Rica en el Science Citation Index: análisis bibliométrico del trienio 1999-2001. Revista de Biología Tropical, 50(3-4), 951-962.

Michán, L. \& Llorente-Bousquets (2010). Bibliometría de la sistemática biológica sobre América Latina durante el siglo XX en tres bases de datos mundiales. Revista de Biología Tropical, 58, 531-545.

Monge-Nájera J., \& Díaz, L. (1988). Thirty-five years of Tropical biology: a quantitative history. Revista de Biología Tropical, 36, 347-359.

Monge-Nájera, J., \& Nielsen-Muñoz, V. (2005). The countries and languages that dominate biological research at the beginning of the 21 st century. Revista de Biología Tropical, 53(1-2), 283-294.

Nielsen-Muñoz, V., Azofeifa-Mora, A., \& Monge-Nájera, J. (2012). Bibliometry of Costa Rica biodiversity studies published in the Revista de Biología Tropical (2000-2010): the content and importance of a leading tropical biology journal in its $60^{\text {th }}$ Anniversary. Revista de Biología Tropical, 60, 1405-1413.

Persson, O., Glänzel, W., \& Dannell, R. (2004). Inflationary bibliometric values: the role of scientific collaboration and the need of relative indicators in evaluative studies. Scientometrics, 60, 421-432.

Pravdic, N., \& Olluic-Vucovic, V. (1991). Distribution of scientific productivity: ambiguities in the assignment of author rank. Scientometric, 20(1), 131-144.

Red Iberoamericana de Indicadores e Ciencia y Tecnología (RICYT). (2014). Indicadores. Buenos Aires, Argentina: RICYT. Recuperado de http://www.ricyt. org/indicadores

Ríos-Hilario, A. B. \& Travieso-Rodríguez, C. (2013). Estudio de la Revista Española de Documentación Científica: análisis comparativo de los períodos 1989-1999 y 2000-2010. Revista Española de Documentación Cientifica, 36(3).

Rodríguez-Yunta, L. (2010). Las revistas iberoamericanas en Web of Science y Scopus: Visibilidad internacional 
e indicadores de calidad (Seminario Hispano-Mexicano de Investigación en Bibliotecología y Documentación). México. Recuperado de http://eprints.rclis. org/14490/1/LuisRY7Encuentro.pdf

Sanz-Casado, E., De Filippo D., \& Marugán, S. (2014). Informe sobre los hábitos de publicación de los investigadores de la Universidad de Costa Rica. Análisis de las publicaciones en Web of Science (2003-2012). Madrid, España: INAECU.

Sanz-Valero, J., Tomás-Casterá, V., \& Tomás-Gorriz, V. (2014). Estudio bibliométrico de producción y consumo de la revista Farmacia Hospitalaria (2004-2012). Farmacia Hospitalaria, 38(1), 1-8. doi.org/10.7399/ FH.2014.38.1.1153
Thomson-Reuters. (2014a). Essential Science Indicators.

Thomson-Reuters. (2014b). Journal Citation Report. SCI Edition.

Van Raan, A. (1998). The influence of international collaboration on the impact of research results: Some simple mathematical considerations concerning the role of selfcitations. Scientometrics, 42(3), 423-428.

Wagner-Döbler, R, (2001). Continuity and discontinuity of collaboration behavior since 1800 -from a bibliometric point of view. Scientometrics, 52, 503-517.

Wagner, C., \& Leydesdorff, L. (2005). Network structure, self-organization, and the growth of International collaboration in science. Research Policy, 34, 1608-1618.

\section{See Digital Appendix / Ver Apéndice digital}

